IJMMS 30:1 (2002) 25-29

PII. S0161171202007780

http://ijmms.hindawi.com

(c) Hindawi Publishing Corp.

\title{
BOUNDEDNESS AND MONOTONICITY OF PRINCIPAL EIGENVALUES FOR BOUNDARY VALUE PROBLEMS WITH INDEFINITE WEIGHT FUNCTIONS
}

\author{
G. A. AFROUZI
}

Received 24 June 2001

\begin{abstract}
We study the principal eigenvalues (i.e., eigenvalues corresponding to positive eigenfunctions) for the boundary value problem: $-\Delta u(x)=\lambda g(x) u(x), x \in D ;(\partial u / \partial n)(x)+$ $\alpha u(x)=0, x \in \partial D$, where $\Delta$ is the standard Laplace operator, $D$ is a bounded domain with smooth boundary, $g: D \rightarrow \mathbb{R}$ is a smooth function which changes sign on $D$ and $\alpha \in \mathbb{R}$. We discuss the relation between $\alpha$ and the principal eigenvalues.
\end{abstract}

2000 Mathematics Subject Classification: 35J15, 35J25.

1. Introduction. We investigate the property of principal eigenvalues for the boundary value problem

$$
\begin{gathered}
-\Delta u(x)=\lambda g(x) u(x), \quad x \in D, \\
\frac{\partial u}{\partial n}(x)+\alpha u(x)=0, \quad x \in \partial D,
\end{gathered}
$$

where $D$ is a bounded region in $\mathbb{R}^{N}$ with smooth boundary, $g: D \rightarrow \mathbb{R}$ is a smooth function which changes $\operatorname{sign}$ on $D$ and $\alpha \in \mathbb{R}$.

Such problems have been studied in recent years since Fleming [4] studied the following associated nonlinear problems arising in the study of population genetics:

$$
u_{t}(x, t)=\Delta u+\lambda g(x) f(u), \quad x \in D,
$$

where $f$ is some function of class $C^{1}$ such that $f(0)=0=f(1)$.

Fleming's results suggested that nontrivial steady-state solutions were bifurcating the trivial solutions $u \equiv 0$ and $u \equiv 1$. In order to investigate these bifurcation phenomena, it was necessary to understand the eigenvalues and eigenfunctions of the corresponding linearized problem

$$
-\Delta u(x)=\lambda g(x) u(x), \quad x \in D .
$$

The study of the linear ordinary differential equation case, however, goes back to Bocher [3]. Attention has been confined mainly to the cases of Dirichlet $(\alpha=\infty)$ and Neumann boundary conditions.

In the case of Dirichlet boundary conditions, it is well known (see [5]) that there exists a double sequence of eigenvalues for (1.1)

$$
\cdots<\lambda_{2}^{-}<\lambda_{1}^{+}<0<\lambda_{1}^{+}<\lambda_{2}^{+}<\cdots,
$$


$\lambda_{1}^{+}\left(\lambda_{1}^{-}\right)$being the unique positive (negative) principal eigenvalue, that is, (1.1) has solution $u(v)$ which is positive in $D$. It is also well known that the case where $0<\alpha<$ $\infty$ is similar to the Dirichlet case.

In the case of Neumann boundary conditions, 0 is clearly a principal eigenvalue and there is a positive (negative) principal eigenvalue if and only if $\int_{D} g(x) d x<0$ $(>0)$; in the case where $\int_{D} g(x) d x=0$ there are no positive and no negative principal eigenvalues.

We show that the set of $\lambda$ 's such that $\lambda$ is a principal eigenvalue of (1.1) is a bounded set and its bounds are independent of $\alpha$, and also the positive principal eigenvalue $\lambda$ of (1.1) is strictly an increasing function of $\alpha$.

Our analysis is based on a method used by Hess and Kato [5]. Consider, for fixed $\lambda$, the eigenvalue problem

$$
\begin{gathered}
-\Delta u(x)-\lambda g(x) u(x)=\mu u(x), \quad x \in D, \\
\frac{\partial u}{\partial n}(x)+\alpha u(x)=0, \quad x \in \partial D .
\end{gathered}
$$

We denote the lowest eigenvalue of (1.5) by $\mu(\alpha, \lambda)$. Let

$$
A_{\alpha, \lambda}=\left\{\int_{D}|\nabla \phi|^{2} d x+\alpha \int_{\partial D} \phi^{2} d s_{x}-\lambda \int_{D} g \phi^{2} d x: \phi \in W^{1,2}(D), \int_{D} \phi^{2} d x=1\right\}
$$

when $\alpha \geq 0$, it is clear that $A_{\alpha, \lambda}$ is bounded below. It is shown in [6], by using variational arguments, that $\mu(\alpha, \lambda)=\inf A_{\alpha, \lambda}$ and that an eigenfunction corresponding to $\mu(\alpha, \lambda)$ does not change sign on $D$. Thus, clearly, $\lambda$ is a principal eigenvalue of (1.1) if and only if $\mu(\alpha, \lambda)=0$.

When $\alpha<0$, the boundedness below of $A_{\alpha, \lambda}$ is no longer obvious a priori, and it is shown by Afrouzi and Brown [2].

2. Boundedness and monotonicity of principal eigenvalues. The following theorem is proved in [1, Theorem 1.8].

THEOREM 2.1. If

$$
\lambda_{1}=\inf \left\{\int_{D}\left[|\nabla \phi|^{2}+q \phi^{2}\right] d x+\alpha \int_{\partial D} \phi^{2} d s_{x}: \phi \in W^{1,2}(D), \int_{D} \phi^{2} d x=1\right\} \text {, }
$$

where $q \in L^{\infty}(D)$, then there exists $\phi_{1} \in W^{1,2}(D), \int_{D} \phi_{1}^{2} d x=1$, such that

$$
\lambda_{1}=\int_{D}\left[\left|\nabla \phi_{1}\right|^{2}+q \phi_{1}^{2}\right] d x+\alpha \int_{\partial D} \phi_{1}^{2} d s_{x} .
$$

Moreover, $\lambda_{1}$ is the principal eigenvalue and $\phi_{1}>0$ is a principal eigenfunction of

$$
\begin{gathered}
-\Delta u(x)+q(x) u(x)=\lambda u(x), \quad x \in D, \\
\frac{\partial u}{\partial n}(x)+\alpha u(x)=0, \quad x \in \partial D .
\end{gathered}
$$


It is obvious that $\lambda_{1}$ is the principal eigenvalue of (1.1) if and only if 0 is the principal eigenvalue of

$$
\begin{gathered}
-\Delta u(x)-\lambda_{1} g(x) u(x)=\mu u(x), \quad x \in D, \\
\frac{\partial u}{\partial n}(x)+\alpha u(x)=0, \quad x \in \partial D .
\end{gathered}
$$

Here we are ready to prove one of the main results of this section about the uniformly boundedness of principal eigenvalues of (1.1) with respect to $\alpha$.

THEOREM 2.2. There exist $m<0$ and $M>0$ such that if $\lambda$ is a principal eigenvalue of (1.1), then $\lambda \in[m, M]$ and also $m, M$ are independent of $\alpha$.

Proof. Suppose that $\lambda_{1}$ is a principal eigenvalue of (1.1). Then 0 is a principal eigenvalue of (2.4) and so by Theorem 2.1, we have

$$
0=\inf \left\{\int_{D}|\nabla \phi|^{2}+\alpha \int_{\partial D} \phi^{2} d s_{x}-\lambda_{1} \int_{D} g \phi^{2} d x: \phi \in W^{1,2}(D), \int_{D} \phi^{2} d x=1\right\} .
$$

Now, by considering test functions $\phi_{1}, \phi_{2} \in C_{0}^{\infty}(D)$ such that $\int_{D} \phi_{1}^{2} d x=1$ and $\int_{D} g \phi_{1}^{2} d x>0$ also $\int_{D} \phi_{2}^{2} d x=1$ and $\int_{D} g \phi_{2}^{2} d x<0$ we have

$$
0 \leq \int_{D}\left|\nabla \phi_{1}\right|^{2}+\alpha \int_{\partial D} \phi_{1}^{2} d s_{x}-\lambda_{1} \int_{D} g \phi_{1}^{2} d x
$$

and also

$$
0 \leq \int_{D}\left|\nabla \phi_{2}\right|^{2}+\alpha \int_{\partial D} \phi_{2}^{2} d s_{x}-\lambda_{1} \int_{D} g \phi_{2}^{2} d x .
$$

Hence from (2.6) and (2.7) we obtain, respectively,

$$
\lambda_{1} \leq \frac{\int_{D}\left|\nabla \phi_{1}\right|^{2} d x}{\int_{D} g \phi_{1}^{2} d x}, \quad \frac{\int_{D}\left|\nabla \phi_{2}\right|^{2} d x}{\int_{D} g \phi_{2}^{2} d x} \leq \lambda_{1} .
$$

So by assuming $M=\int_{D}\left|\nabla \phi_{1}\right|^{2} d x / \int_{D} g \phi_{1}^{2} d x$ and $m=\int_{D}\left|\nabla \phi_{2}\right|^{2} d x / \int_{D} g \phi_{2}^{2} d x$, we have obtained $\lambda \in[m, M]$, and also we see that $m, M$ are independent of $\alpha$.

In the case $0<\alpha<\infty$, it is known [1, Lemmas 1.18 and 1.19] that problem (1.1) has the unique positive (negative) principal eigenvalue, that is, $\lambda_{1}^{+}\left(\lambda_{1}^{-}\right)$, such that if $u$ and $v$ are being eigenfunctions corresponding to $\lambda_{1}^{+}$and $\lambda_{1}^{-}$, respectively, then $\int_{D} g u^{2} d x>0$ and $\int_{D} g v^{2} d x<0$. Also in the case $\alpha<0$, the following theorem [2, Theorem 5] is proved.

THEOREM 2.3. There exists $\alpha_{0} \leq 0$ such that

(i) if $\alpha<\alpha_{0}$, then (1.1) does not have a principal eigenvalue;

(ii) if $\alpha=\alpha_{0}$, then (1.1) has a unique principal eigenvalue with the corresponding eigenfunction $u_{0}$ such that $\int_{D} g(x) u_{0}^{2}(x) d x=0$;

(iii) if $\alpha>\alpha_{0}$, then (1.1) has exactly two principal eigenvalues $\lambda$ and $\mu, \lambda<\mu$; if $u_{0}$ and $v_{0}$ are eigenfunctions corresponding to $\lambda<\mu$, respectively, then $\int_{D} g(x) u_{0}^{2}(x) d x<0$ and $\int_{D} g(x) v_{0}^{2}(x) d x>0$; 
(iv) $\alpha_{0}=0$ if and only if $\int_{D} g(x) d x=0$.

Now we prove the monotonicity of principal eigenvalues of (1.1) with respect to $\alpha$.

THEOREM 2.4. Suppose that $\lambda_{1}$ is a principal eigenvalue of

$$
\begin{gathered}
-\Delta u(x)=\lambda g(x) u(x), \quad x \in D, \\
\frac{\partial u}{\partial n}(x)+\alpha_{1} u(x)=0, \quad x \in \partial D
\end{gathered}
$$

such that the corresponding principal eigenvalue, say $u_{1}$, satisfies $\int_{D} g u_{1}^{2} d x>0$. If $\alpha_{2}>\alpha_{1}$ and $\lambda_{2}, u_{2}$ are, respectively, principal eigenvalue and eigenfunction of

$$
\begin{gathered}
-\Delta u(x)=\lambda g(x) u(x), \quad x \in D, \\
\frac{\partial u}{\partial n}(x)+\alpha_{2} u(x)=0, \quad x \in \partial D
\end{gathered}
$$

such that $\int_{D} g u_{2}^{2} d x>0$, then $\lambda_{2}<\lambda_{1}$.

Proof. Since $\lambda_{1}$ is a principal eigenvalue of (2.9), so 0 is a principal eigenvalue of

$$
\begin{gathered}
-\Delta u(x)-\lambda_{1} g(x) u(x)=\mu u(x), \quad x \in D, \\
\frac{\partial u}{\partial n}(x)+\alpha_{1} u(x)=0, \quad x \in \partial D,
\end{gathered}
$$

and so we have

$$
0=\int_{D}\left|\nabla u_{1}\right|^{2} d x+\alpha_{1} \int_{\partial D} u_{1}^{2} d s_{x}-\lambda_{1} \int_{D} g u_{1}^{2} d x
$$

and also

$$
0=\inf \left\{\int_{D}|\nabla u|^{2} d x+\alpha_{2} \int_{\partial D} u^{2} d s_{x}-\lambda_{2} \int_{D} g u^{2} d x: u \in W^{1,2}(D), \int_{D} u^{2} d x=1\right\} .
$$

If $\lambda_{2} \geq \lambda_{1}$, then

$$
\begin{aligned}
0 & =\int_{D}\left|\nabla u_{1}\right|^{2} d x+\alpha_{1} \int_{\partial D} u_{1}^{2} d s_{x}-\lambda_{1} \int_{D} g u_{1}^{2} d x \\
& >\int_{D}\left|\nabla u_{1}\right|^{2} d x+\alpha_{2} \int_{\partial D} u_{1}^{2} d s_{x}-\lambda_{2} \int_{D} g u_{1}^{2} d x \\
& \geq 0
\end{aligned}
$$

which is impossible. Hence $\lambda_{2}<\lambda_{1}$ and the proof is complete.

\section{REFERENCES}

[1] G. A. Afrouzi, Some problems in elliptic equations involving indefinite weight functions, Ph.D. thesis, Heriot-watt University, Edinburgh, UK, 1997.

[2] G. A. Afrouzi and K. J. Brown, On principal eigenvalues for boundary value problems with indefinite weight and Robin boundary conditions, Proc. Amer. Math. Soc. 127 (1999), no. $1,125-130$. 
[3] M. Bocher, The smallest characteristic numbers in a certain exceptional case, Bull. Amer. Math. Soc. 21 (1914), 6-9.

[4] W. H. Fleming, A selection-migration model in population genetics, J. Math. Biol. 2 (1975), no. 3, 219-233.

[5] P. Hess and T. Kato, On some linear and nonlinear eigenvalue problems with an indefinite weight function, Comm. Partial Differential Equations 5 (1980), no. 10, 999-1030.

[6] J. Smoller, Shock Waves and Reaction-Diffusion Equations, Springer-Verlag, New York, 1983.

G. A. Afrouzi: Department of Mathematics, Faculty of Basic Sciences, Mazandaran UNIVERSITY, BABOLSAR, IRAN 


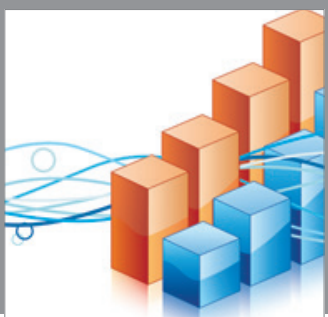

Advances in

Operations Research

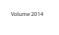

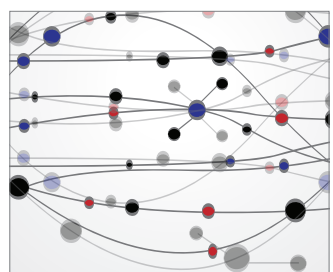

\section{The Scientific} World Journal
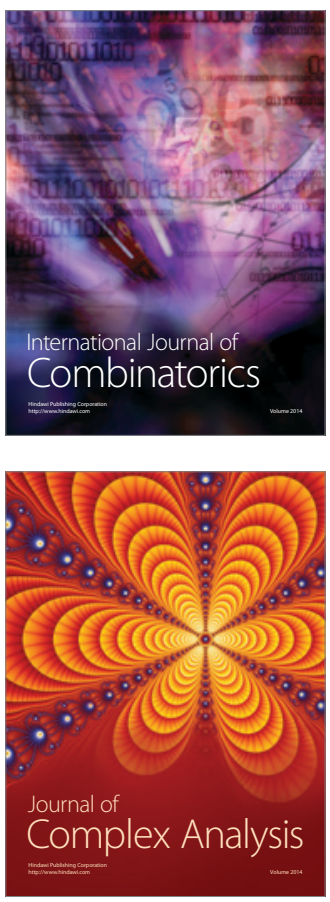

International Journal of

Mathematics and

Mathematical

Sciences
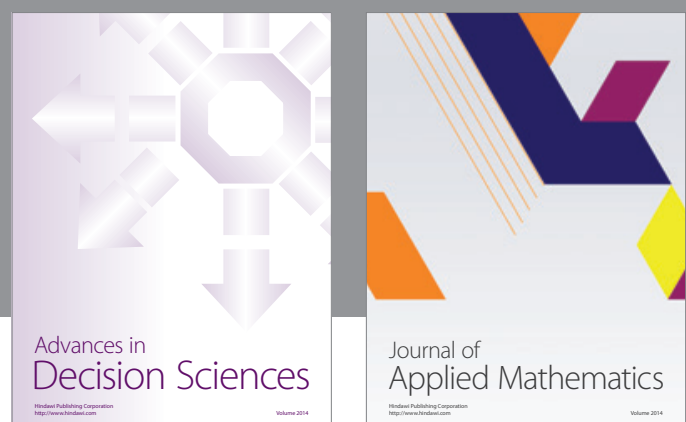

Journal of

Applied Mathematics
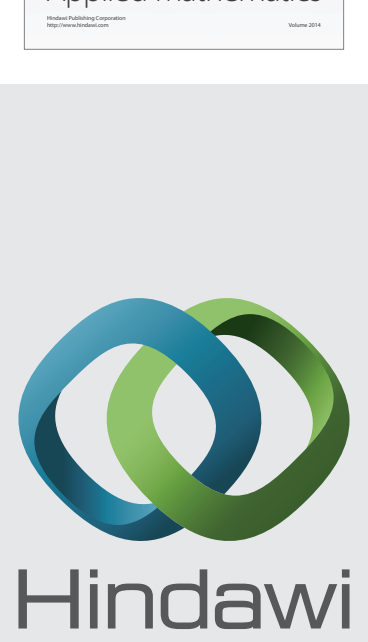

Submit your manuscripts at http://www.hindawi.com
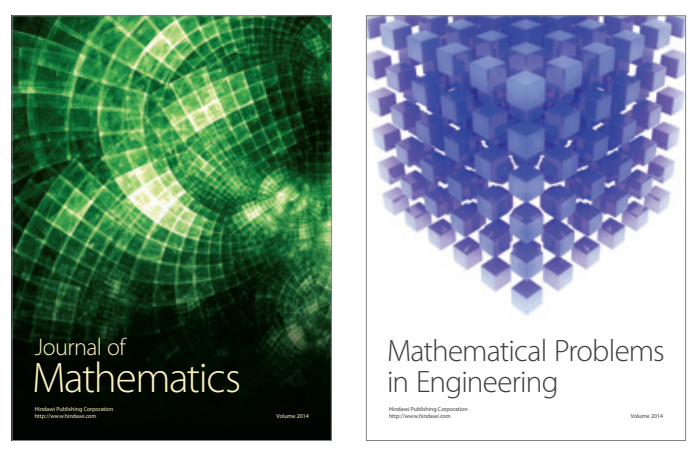

Mathematical Problems in Engineering
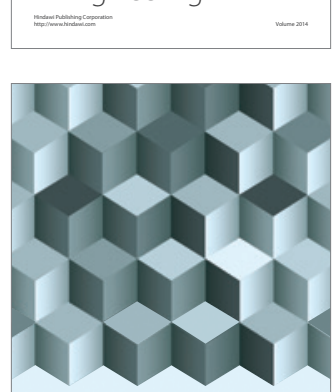

Journal of

Function Spaces
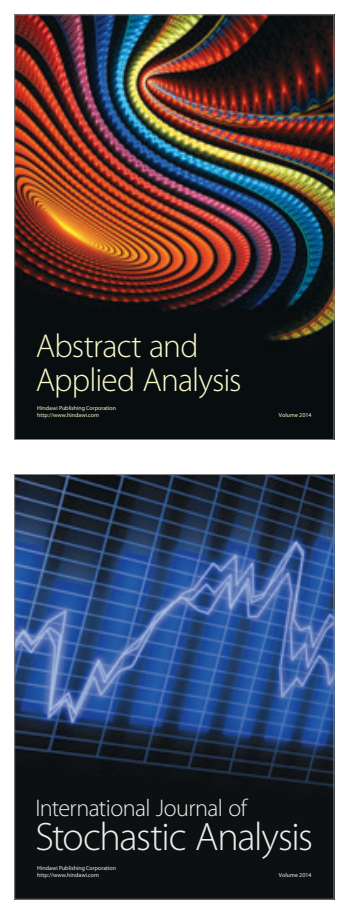

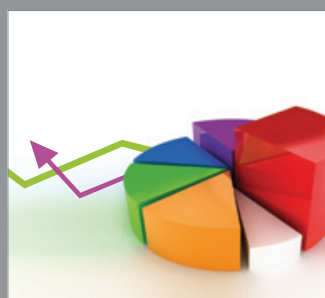

ournal of

Probability and Statistics

Promensencen
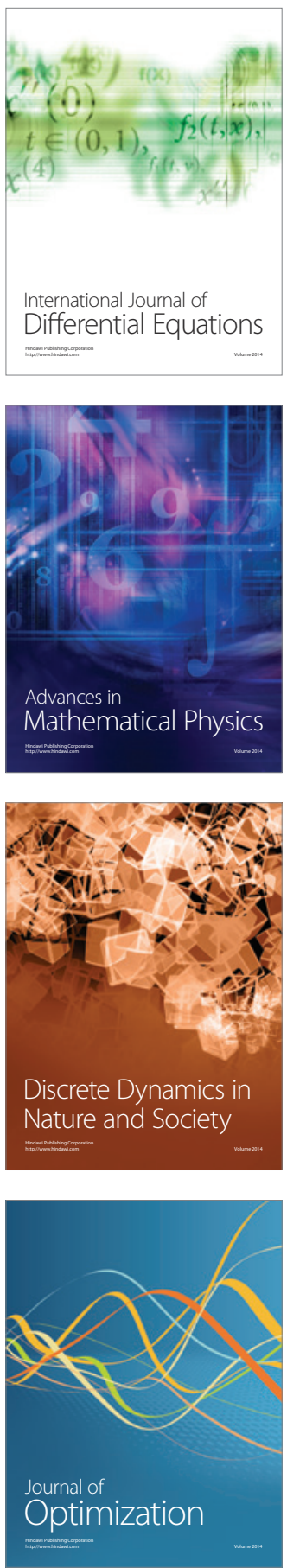